\title{
Do patient reported outcome measures capture actual upper limb recovery?
}

$\mathrm{T}$ he primary focus of rehabilitation following injury to the upper limb is to improve the use of the limb in day-today tasks (Wade, 2003). How we use either limb, however, varies with any given task. While for bimanual tasks the limbs may work symmetrically, asymmetrically or cooperatively, the dominant limb is preferred for precision tasks and is used more frequently during the day than the non-dominant limb (Kilbreath and Heard, 2005). If injury occurs to the dominant limb, the impact on the individual will be markedly different to loss of the non-dominant limb, particularly for those with severe long-term disability who may be forced to change their dominance over time. Furthermore, after very severe trauma, for example, upper limb amputation, peripheral nerve injury or stroke, some individuals will live with the use of only one arm, and rely on their teeth, legs or other parts of their anatomy to stabilise objects.

\section{Measuring outcomes}

Choosing an outcome measure that can accurately assess the impact of upper limb injury from the plethora available is a challenge to clinicians and researchers. In recent years, the World Health Organization (WHO) has provided a conceptual framework, the International Classification of Functioning, Disability and Health (ICF) (WHO, 2011), to differentiate between various aspects of health. In the $I C F$, physiological functions, such as strength, pain or mood, are classified as 'body functions and structures', and the concepts of 'activity' and 'participation' are introduced to cover domains related to functioning.

While physiological functions are important, most people with upper limb injuries will determine their overall outcome based on how well they use their limb to perform day-to-day activi- ties. Therefore, in recent years more and more emphasis has been placed on measuring the impact of a disorder on the individual from their perspective using patient reported outcome measures (PROMs). Indeed, it is not uncommon to see funding bodies providing funding solely on the basis of PROM change scores using PROMs they stipulate.

\section{Are all upper limb tasks relevant to all disorders?}

To measure the function of the upper limb, PROMs need to contain items that reflect how the limb is used; that is, to stabilise, reach, grasp and manipulate objects for basic life skills, such as feeding, dressing, personal care, domestic chores, communication and work (Barreca et al, 2004). When choosing a PROM, many clinicians will consider the evidence related to reliability, validity, and ability to detect change in their target population. However, clinicians also need to consider whether the measure (i.e. the type and difficulty of the items) matches therapeutic goals, and has the scope or capacity to detect and quantify change as the person recovers.

Items contained in a PROM need to be relevant, meaningful and important to the person or group being assessed, and cover the full spectrum of ability from minimal use to typical or "normal' use. While many PROMs seem to contain a very similar set of items, they do not all reflect use of the limb across a range of disorders. For example, the ABILHAND was developed as a PROM of manual ability for individuals with rheumatoid arthritis, regardless of the strategy used to perform the tasks (Penta et al, 2001). There are now six diagnostic-specific versions of the ABILHAND, and each version was developed using a slightly different set of items deemed to be representative of the disorder being measured.

A generic ABILHAND has recently been developed (Arnould et al, 2012).
However, only eleven items share a common difficulty across all diagnoses determined by the underlying disorder. For example, bimanual activities, such as 'peeling potatoes with a knife', were found to be more difficult for individuals with an asymmetric disorder, while activities that can be performed using one hand or bimanual activities that can be completed in phases using only one hand, such as 'use a stapler', were easier for those with an asymmetric disorder as respondents used their unaffected limb to perform the task.

In contrast, those with a proximal upper limb injury found activities that required greater shoulder range more difficult, while those with a disorder affecting the hand found activities that needed fine finger control more difficult. Moreover, the authors found the generic version not as sensitive to change or able to identify people with different levels of ability as the original disease-specific versions. These results seem to indicate that different activities are required to accurately reflect both the nature of the disorder and region of the limb affected. One generic list of activities may not necessarily apply to all conditions.

\section{Can PROMs assess more than one factor at once?}

A number of dimensions or factors (physiological functions, such as strength or pain), activity limitations or participation restrictions may be affected following injury to the upper limb. Generic measures have been developed that combine a number of these factors. However, measuring different factors and combining them to produce a single composite score can be problematic.

For example, the Disability of Arm Shoulder and Hand (DASH) (Hudak et al, 1996) was designed to measure a single factor termed 'symptoms and physical function'. However, recent statistical analyses have shown the DASH 
to contain a number of different factors, all measuring different aspects of upper limb use. Franchignoni et al (2010) identified three factors they called 'shoulder range of motion', 'manual functioning' and 'symptoms' (classified as 'body functions' in the $I C F)$. Lehman et al (2011) also identified three factors they termed 'gross motor activities requiring whole body movements', 'fine motor activities' and 'symptoms'. Thus, change as measured by the DASH may occur as a result of improved use of the arm to perform day-to-day tasks. However, change could equally be due to alteration in 'body function' domains, such as better sleep, less pain or improved mood, with no actual improvement in day-today use of the arm. Further, summed scores from measures that are known to be made up of a number of factors should be viewed with extreme caution (Packham and MacDermid, 2013) and draw into question whether they measure what they purport to measure.

\section{Should PROMs be limb-specific?}

Both the ABILHAND and DASH ask respondents to answer items regardless of the strategy used, i.e. they do not differentiate between use of the affected or unaffected limb to perform tasks. Therefore, it is difficult to determine whether results reflect the impact of the injury on the person and whether a specific intervention has led to improved day-to-day use of the affected limb. Alternatively, these PROMs may actually measure the ability to compensate with the unaffected limb, particularly for those with long-term disability and very limited upper limb use.

For example, when responding to a unimanual task, such as 'turning a key', two people with the same unilateral upper limb condition may respond differently. The person with a flail limb following complete brachial plexus injury may answer 'with great difficulty' as they are unable to perform the task with their injured limb, while the second person may respond 'no difficulty' as they can easily perform the task with their unaffected arm. Both responses are correct as the question does not specify that they have to respond in relation to their affected or unaffected limb. If, over time, the person who responded 'with great difficulty' adopts compensatory techniques or changes their dominance, it is conceivable that they may respond 'no difficulty' on reassessment. This change would indicate significant improvement, yet their affected upper limb may remain flail.

If the response is not targeted to the affected upper limb, PROM change scores may indicate recovery when, in fact, none exists. Some PROMs, such as the Upper Extremity Functional Index (Stratford et al, 2001) and the Patient-Rated Wrist and Hand Evaluation (MacDermid, 1996), circumvent the issue of limb use by asking for responses only as they relate to the injured limb. However, recent statistical analyses have shown both to have many of the same issues as the PROMs discussed above, including the presence of multiple factors (Hamilton and Chesworth, 2013; Packham and MacDermid, 2013).

It could be argued that PROMs are only meant to assess changes in ability over time, regardless of how this is achieved. But what does this mean for clinicians who target their management to the affected limb and who wish to know whether their therapeutic input has resulted in change to that limb? If, for example, clinicians wish to compare two therapeutic techniques, it is essential to ascertain whether the measured change can be attributed to the intervention and has not occurred as a result of compensation with the uninjured limb, alteration of dominance, or another factor completely unrelated to actual use of the limb.

\section{CONCLUSIONS}

As greater emphasis is placed on the use of PROMs, it is essential that clinicians understand what is actually being measured in order to monitor progress or identify change, to compare treatment effectiveness, or to undertake a cost-benefit analysis specific to the population of interest. As funding for health care becomes more and more linked to quantifiable outcomes, educators, researchers and clinicians must be fully aware of the strengths and limitations of the outcome measures they use, and be closely involved in the development and selection of appropriate tools that truly reflect their client's capacity. IJTR

Arnould C, Vandervelde L, Batcho CS, Penta M, Thonnard JL (2012) Can manual ability be measured with a generic ABILHAND scale? A cross-sectional study conducted on six diagnostic groups. BMJ Open 2(6): e001807

Barreca S, Gowland CK, Stratford P et al (2004) Development of the Chedoke Arm and Hand Activity Inventory: theoretical constructs, item generation, and selection. Top Stroke Rehabil 11(4): 31-42

Franchignoni F, Giordano A, Sartorio F et al (2010) Suggestions for refinement of the Disabilities of the Arm, Shoulder and Hand Outcome Measure (DASH): a factor analysis and Rasch validation study. Arch Phys Med Rehabil 91(9): 1370-7

Hamilton CB, Chesworth BM (2013) A Raschvalidated version of the upper extremity functional index for interval-level measurement of upper extremity function. Phys Ther 93(11) 1507-19

Hudak PL, Amadio PC, Bombardier C (1996) Development of an upper extremity outcome measure: the DASH (disabilities of the arm, shoulder and hand) [corrected]. Am J Ind Med 29(6): 602-8

Kilbreath SL, Heard RC (2005) Frequency of hand use in healthy older persons. Aust $J$ Physiother 51(2): 119-22

Lehman LA, Woodbury M, Velozo CA (2011) Examination of the factor structure of the Disabilities of the Arm, Shoulder, and Hand questionnaire. Am J Occup Ther 65(2): 169-78

MacDermid JC (1996) Development of a scale for patient rating of wrist pain and disability. $J$ Hand Ther 9(2): 178-83

Packham T, MacDermid JC (2013) Measurement properties of the Patient-Rated Wrist and Hand Evaluation: Rasch analysis of responses from a traumatic hand injury population. J Hand Ther 26(3): 216-23

Penta M, Tesio L, Arnould C, Zancan A, Thonnard JL (2001) The ABILHAND questionnaire as a measure of manual ability in chronic stroke patients: Rasch-based validation and relationship to upper limb impairment. Stroke 32(7): 1627-34

Stratford PW, Binkley JM, Stratford DM (2001) Development and initial validation of the Upper Extremity Functional Index. Physiother Can 53: 259-67

Wade DT (2003) Outcome measures for clinical rehabilitation trials: impairment, function, quality of life, or value? Am J Phys Med Rehabil 82(10 Suppl): S26-31

World Health Organization (2001) International Classification of Functioning, Disability and Health $(I C F)$. WHO, Geneva

Bridget Hill is a PhD candidate, School of Allied Health Sciences, Griffith University, Queensland, Australia;

Andrea Bialocerkowski is deputy head of learning and teaching, School of Allied

Health Sciences, Griffith University,

Queensland, Australia;

Gavin Williams is a specialist neurological

physiotherapist and research fellow,

Epworth Hospital, Melbourne, Australia. 
Copyright of International Journal of Therapy \& Rehabilitation is the property of Mark Allen Publishing Ltd and its content may not be copied or emailed to multiple sites or posted to a listserv without the copyright holder's express written permission. However, users may print, download, or email articles for individual use. 